\title{
Integrable Cases of a Rigid Body Dynamics and Integrable Systems on the Ellipsoids
}

\author{
O. I. Bogoyavlensky \\ V. A. Steklov Mathematical Institute, Academy of Sciences of the USSR, \\ SU-117966 Moscow, USSR
}

\begin{abstract}
Infinite-dimensional sets of integrable cases are found for the equations of a rigid body rotation around a fixed point in an axially symmetric potential field and also in more complicated fields in the presence of some symmetry of the rigid body inertia tensor.
\end{abstract}

\section{Introduction}

Rotation of a rigid body around a fixed point in some potential field is described by the Lagrangian system determined on the Lie group $\mathrm{SO}(3)$ with the Lagrangian

$$
L=\frac{1}{2}\left(I_{1} \omega_{1}^{2}+I_{2} \omega_{2}^{2}+I_{3} \omega_{3}^{2}\right)-V\left(Q_{j}^{i}\right) .
$$

Here $I_{k}$ are the eigenvalues of the inertia tensor, $\omega$ is the angular velocity vector, with components $\omega_{k}, Q$ is the orthogonal matrix, with elements $Q_{j}^{i}$, determining the position of the rigid body, $V\left(Q_{j}^{i}\right)$ is a potential function. The classical integrable cases of a rigid body dynamics [1] have the following common properties: firstly all of them depend on some finite and not great number of parameters, determining the special forms of the potential function $V\left(Q_{j}^{i}\right)$ and special values of the inertia tensor components $I_{k}$, and secondly the dynamics of trajectories of the corresponding integrable Hamiltonian systems is a linear winding of two-dimensional invariant tori $\mathbb{T}^{2}$.

The present work is devoted to construction of new cases of integrability for the equations of a rigid body dynamics, depending on an infinite number of parameters which determine the form of the potential function $V\left(Q_{j}^{i}\right)$. A number of geometrical methods are used connected with the existence of the maps

$$
S^{3} \stackrel{f}{\longrightarrow} \mathrm{SO}(3) \stackrel{g}{\longrightarrow} S^{2} .
$$

The map $f$ is the universal covering, the map $g$ is the fibration, its fibres are circumferences $S^{1} ; S^{n}$ is an $n$-dimensional sphere. The composition of the maps $g \circ f$ is the known Hopf fibration. Due to the presence of the maps $f$ and $g(1.2)$, it is 
possible to connect the integrable Lagrangian systems on the Lie group $\mathrm{SO}(3)$ with the integrable Lagrangian systems on the spheres $S^{3}$ and $S^{2}$.

There are two classical integrable systems on the spheres $S^{n}$ : the Jacobi geodesic flow on the ellipsoid $[2,3]$ (and also under the influence of a linear force of some special form) and the Neumann mass point dynamics on the sphere $S^{n}$ in a potential field with an arbitrary quadratic potential [4], for modern development see [5-8]. An infinite-dimensional set of integrable Lagrangian systems on the sphere $S^{2}$, generalizing the Neumann system, was found in [21]. In recent preprints $[33,34]$ a "recursive description" was done for infinite families of integrable potentials on $n$-dimensional quadrics. In Sect. 3 of the present work we obtain the explicit formulae for the integrable potentials (which may be polynomials of an arbitrary even degree or entire functions), having important applications for $n=2, n=3$ in a rigid body dynamics, see Eqs. (3.27), (4.21), (5.8).

New integrable cases of a rigid body dynamics, constructed in the present work, are found on the base of three different constructions connected with the maps (1.2), for three types of the rigid body inertia tensor: spherical $\left(I_{1}=I_{2}=I_{3}\right)$, symmetric $\left(I_{1}=I_{2} \neq I_{3}\right)$, and an arbitrary one $\left(I_{1} \neq I_{2} \neq I_{3}\right)$.

\section{Rotation of an Axially Symmetric Rigid Body in a Newtonian Field of an Arbitrary Remote Object}

In [10] the theorem was proved about the integrability in Liouville's sense of a rigid body rotation around fixed center of mass in a Newtonian field of an arbitrary remote object. Also the explicit formulae were obtained [11] describing the rigid body rotation in the terms of the Riemannian theta-functions of four variables $\theta\left(z_{1}, z_{2}, z_{3}, z_{4}\right)$, restricted on the Prym variety. In this section we show that for an axially symmetric rigid body $\left(I_{1}=I_{2}\right)$ the new integrable case $[10,11]$ is connected with the classical Neumann system on the sphere $S^{2}[4]$ and with Clebsch's case of integrability [12] for the Kirchhoff equations. Because of this connection it is possible to describe the dynamics of the axially symmetric rigid body in terms of Riemannian theta-functions of two variables $\theta\left(z_{1}, z_{2}\right)$.

The problem under investigation is equivalent to the study of a rigid body rotation around a fixed point in a Newtonian field with an arbitrary homogeneous quadratic potential, which may be reduced to the diagonal form

$$
\varphi=\frac{1}{2}\left(a_{1} x_{1}^{2}+a_{2} x_{2}^{2}+a_{3} x_{3}^{2}\right) .
$$

Here $x_{1}, x_{2}, x_{3}$ are the coordinates in the resting system of reference $F$. Let $S$ be a moving reference frame related to the rigid body, its basis vectors coincide with the principal axes of the rigid body inertia tensor, so $I_{i k}=I_{k} \delta_{i k}$. Denote $\boldsymbol{\alpha}, \boldsymbol{\beta}, \boldsymbol{\gamma}$ the unit basis vectors of the resting reference frame $F$, as given in the moving frame $S$. The Lagrangian (1.1) takes the form

$$
\begin{aligned}
2 L= & I_{1} \omega_{1}^{2}+I_{2} \omega_{2}^{2}+I_{3} \omega_{3}^{2}-a_{1}\left(I_{1} \alpha_{1}^{2}+I_{2} \alpha_{2}^{2}+I_{3} \alpha_{3}^{2}\right) \\
& -a_{2}\left(I_{1} \beta_{1}^{2}+I_{2} \beta_{2}^{2}+I_{3} \beta_{3}^{2}\right)-a_{3}\left(I_{1} \gamma_{1}^{2}+I_{2} \gamma_{2}^{2}+I_{3} \gamma_{3}^{2}\right) .
\end{aligned}
$$

We denote $\mathbf{q}(t)$ the unit vector along the symmetry axis of the rigid body inertia tensor (corresponding to the eigenvalue $I_{3}$ ). Position of the rigid body in the frame 
$F$ is determined by three Euler angles $\theta, \psi, \varphi, \theta$ is the angle between vectors $\mathbf{q}(t)$ and $\boldsymbol{\beta}$ (the nutation angle), $\psi$ is the precession angle, $\varphi$ is the angle of the rotation of the rigid body around the symmetry axis $\mathbf{q}(t)$. The vector $\mathbf{q}(t)$ has the following coordinates in the resting frame $F$ :

$$
q_{1}=\sin \theta \sin \psi, \quad q_{2}=-\sin \theta \cos \psi, \quad q_{3}=\cos \theta .
$$
form

The Lagrangian function (2.1) for an axially symmetric rigid body acquires the

$$
L_{1}=\frac{1}{2} I_{1}\left(\dot{\theta}^{2}+\dot{\psi}^{2} \sin ^{2} \theta\right)+\frac{1}{2} I_{3}(\dot{\psi} \cos \theta+\dot{\varphi})^{2}-V(\mathbf{q})
$$

In consequence of Eqs. (2.1)-(2.3) the Lagrangian $L_{1}$ does not depend on the angle $\varphi$, therefore the corresponding momentum $p_{\varphi}$ is the first integral of the Lagrangian system

$$
p_{\varphi}=\partial L_{1} / \partial \dot{\varphi}=I_{3}(\dot{\psi} \cos \theta+\dot{\varphi}) .
$$

The Routh function $L_{2}=L_{1}-p_{\varphi} \dot{\varphi}$, written in coordinates of the vector $\mathbf{q}(t)$ $((\mathbf{q}, \mathbf{q})=1)$ has the form

$$
L_{2}=\frac{1}{2} I_{1}(\dot{\mathbf{q}}, \dot{\mathbf{q}})+p_{\varphi} \dot{\psi} \cos \theta-\frac{1}{2}(\mathbf{q}, A \mathbf{q})-\frac{1}{2 I_{3}} p_{\varphi}^{2},
$$

where the matrix elements of $A$ are $A_{i j}=\left(I_{3}-I_{1}\right) a_{i} \delta_{i j}$. The Lagrangian $L_{2}$ for $p_{\varphi}=0$ coincides with the Lagrangian of the Neumann system [4] on the sphere $S^{2}{ }^{1}$ In the general case $p_{\varphi} \neq 0$, the corresponding Lagrangian equations have the form

$$
I_{1} \ddot{\mathbf{q}}=-A \mathbf{q}-p_{\varphi}(\dot{\mathbf{q}} \times \mathbf{q})+\lambda \mathbf{q},
$$

where the multiplier $\lambda$ is determined so that

$$
\frac{d^{2}}{d t^{2}}(\mathbf{q}, \mathbf{q})=0, \quad \lambda=(A \mathbf{q}, \mathbf{q})-I_{1}(\dot{\mathbf{q}}, \dot{\mathbf{q}}) \text {. }
$$

The term $-p_{\varphi}(\dot{\mathbf{q}} \times \mathbf{q})$ in Eq. (2.6) describes the forces, analogous to the Dirac monopole action [13] on the electric charge and arises from variation of the term $p_{\varphi} \dot{\psi} \cos \theta$ in the Lagrangian (2.5). The investigation of the "generalized Dirac monopoles" arising from the study of Lagrangian structure of the Kirchhoff equations, led to the discovery in the works $[14,15]$ of multivalued functions and multivalued analogy of the Morse theory.

Integrability of Eq. (2.6) in Liouville's sense and in terms of the Riemannian theta-functions of two variables $\theta\left(z_{1}, z_{2}\right)$ on the level $p_{\varphi}=0$ follows from that for the classical Neumann problem [4]. For $p_{\varphi} \neq 0$, Eqs. (2.6) coincide with the equations, arising in the completely different problem - the dynamics of the travelling waves of the magnetization vector in the Landau-Lifshitz equation. These equations are shown [16] to be equivalent to the Clebsch case of integrability for the Kirchhoff equations. Equations (2.6) by the map

$$
\dot{\mathbf{q}} \rightarrow \mathbf{M}=\dot{\mathbf{q}} \times \mathbf{q}-I_{1}^{-1} p_{\varphi} \mathbf{q}
$$

are transformed to the equations

$$
\dot{\mathbf{M}}=\mathbf{q} \times I_{1}^{-1} A \mathbf{q}, \quad \dot{\mathbf{q}}=\mathbf{q} \times \mathbf{M},
$$

1 Therefore the investigated system has also homoclinic orbits [31] 
which describe the special Clebsch integrable case on the surface level of the first integral $(\mathbf{M}, \mathbf{q})=-I_{1}^{-1} p_{\varphi}$. We derive the explicit formulae describing dynamics of the system (2.8) in terms of the Riemannian theta-functions $\theta\left(z_{1}, z_{2}\right)[17,18]$. The Hamiltonian $\mathrm{H}$ and the Clebsch integral $J_{4}$ have the form

$$
\begin{gathered}
2 H=(\mathbf{M}, \mathbf{M})+\left(\mathbf{q}, I_{1}^{-1} A \mathbf{q}\right), \\
J_{4}=\left(\mathbf{M}, I_{1}^{-1} A \mathbf{M}\right)-I_{1}^{-3} A_{1} A_{2} A_{3}\left(\mathbf{q}, I_{1} A^{-1} \mathbf{q}\right) .
\end{gathered}
$$

We denote $s_{1}, s_{2}, s_{3}, s_{4}$ the roots of the equation $f(s)=0$

$f(s)=\left[s^{2}-s\left(\operatorname{Tr}\left(I_{1}^{-1} A\right)-2 H\right)-J_{4}\right]^{2}-4 I_{1}^{-2} p_{\varphi}^{2}\left(s-I_{1}^{-1} A_{1}\right)\left(s-I_{1}^{-1} A_{2}\right)\left(s-I_{1}^{-1} A_{3}\right)$.

We introduce the Riemannian surface $\Gamma$ determined by the equation

$$
y^{2}=P(z), \quad P(z)=z\left(z-d_{1}^{2}\right)\left(z-d_{2}^{2}\right)\left(z-d_{3}^{2}\right)\left(z-d_{1}^{2} d_{2}^{2} d_{3}^{2}\right),
$$

where the parameters $d_{1}, d_{2}, d_{3}$ are defined by the equalities

$$
d_{k}=\left(\sqrt{\frac{s_{3}-I_{1}^{-1} A_{k}}{f^{\prime}\left(s_{3}\right)}}+i \sqrt{\frac{s_{4}-I_{1}^{-1} A_{k}}{f^{\prime}\left(s_{4}\right)}}\right)\left(\sqrt{\frac{s_{1}-I_{1}^{-1} A_{k}}{f^{\prime}\left(s_{1}\right)}}+i \sqrt{\frac{s_{2}-I_{1}^{-1} A_{k}}{f^{\prime}\left(s_{2}\right)}}\right)^{-1} .
$$

The genus of the surface $\Gamma$ is $g(\Gamma)=2$. Formulae [18], describing the dynamics of trajectories of the system (2.8) look as follows

$$
q_{k}=i_{k} \frac{\theta\left[r_{k}\right]\left(\mathbf{z}_{0}+\mathbf{U} t\right) \theta\left[m_{k}\right]\left(\mathbf{w}_{0}\right)-\theta\left[r_{k}\right]\left(\mathbf{w}_{0}\right) \theta\left[m_{k}\right]\left(\mathbf{z}_{0}+\mathbf{U} t\right)}{\theta\left[n_{k}\right]\left(\mathbf{z}_{0}+\mathbf{U} t\right) \theta\left[p_{k}\right]\left(\mathbf{w}_{0}\right)-\theta\left[n_{k}\right]\left(\mathbf{w}_{0}\right) \theta\left[p_{k}\right]\left(\mathbf{z}_{0}+\mathbf{U} t\right)} .
$$

Here the theta-functions $\theta[x]\left(z_{1}, z_{2}\right)$ and the vector $\mathbf{U}$ are determined by the Riemannian surface $\Gamma$, the characteristics $r_{k}, m_{k}, n_{k}, p_{k}$ depend on mutual positions of the roots of the polynomial (2.10), constant vectors $z_{0}, w_{0}$ are determined by initial conditions, see [18].

The formulae (2.12) determine the Euler angles $\theta, \psi$ dependence upon time $t$ due to Eqs. (2.2). One can find the Euler angle $\varphi$ dependence upon time $t$ integrating Eq. (2.4).

\section{Integrable Lagrangian Systems in the Euclidean Space, on the $\boldsymbol{n}$-Dimensional Ellipsoids and Spheres $\boldsymbol{S}^{\boldsymbol{n}}$}

1. Let us consider a family [33] of the Lagrangian systems in the Euclidean space $R^{n+1}$ with the Lagrangians of the form

$$
L=\frac{1}{2} \sum_{i=0}^{n}\left(\dot{x}_{i}\right)^{2}-V\left(x_{0}, \ldots, x_{n}\right)
$$

which admit the separation of variables in the Hamilton-Jacobi equation after transformation to the elliptic coordinates and therefore are integrable in quadratures. The elliptic coordinates $u_{0}, \ldots, u_{n}$ in $R^{n+1}$ are defined from the equation [2],

$$
\sum_{v=0}^{n} \frac{x_{v}^{2}}{z+a_{v}}-1=-\frac{U_{0}(z)}{A(z)}, U_{0}(z)=\prod_{j=0}^{n}\left(z-u_{j}\right), A(z)=\prod_{v=0}^{n}\left(z+a_{v}\right),
$$


where $a_{0}, \ldots, a_{n}$ are some constants $\left(a_{i} \neq a_{j}\right)$ and $z$ is an arbitrary parameter. After reduction to the common denominator $A(z)$ and equating the coefficients at $z^{n+1-k}(k=1, \ldots, n+1)$ in both-hand sides of Eq. (3.2), we receive the relations

$$
\begin{gathered}
\sigma_{k}(u)=(-1)^{k}\left(\sigma_{k}(a)-Q_{k-1}\left(x_{0}, \ldots, x_{n}\right)\right), \\
Q_{k}\left(x_{0}, \ldots, x_{n}\right)=\sum_{v=0}^{n} x_{v}^{2}\left(\sigma_{k}(a)+\sum_{i=1}^{k}(-1)^{i} a_{v}^{i} \sigma_{k-i}(a)\right) .
\end{gathered}
$$

Here $\sigma_{k}(y)$ is the elementary symmetric function of variables $y$ of degree $k$, $\sigma_{0}(y) \equiv 1$. In particular we find from Eqs. (3.3),

$$
\begin{gathered}
Q_{0}(x)=x_{0}^{2}+\ldots+x_{n}^{2}, \quad Q_{1}(x)=\sigma_{1}(a) \sum_{v=0}^{n} x_{v}^{2}-\sum_{v=0}^{n} a_{v} x_{v}^{2}, \\
Q_{2}(x)=\sigma_{2}(a) \sum_{v=0}^{n} x_{v}^{2}-\sigma_{1}(a) \sum_{\nu=0}^{n} a_{v} x_{v}^{2}+\sum_{\nu=0}^{n} a_{v}^{2} x_{v}^{2}, \\
Q_{3}(x)=\sigma_{3}(a) \sum_{v=0}^{n} x_{v}^{2}-\sigma_{2}(a) \sum_{v=0}^{n} a_{v} x_{v}^{2}+\sigma_{1}(a) \sum_{v=0}^{n} a_{v}^{2} x_{v}^{2}-\sum_{v=0}^{n} a_{v}^{3} x_{v}^{2} .
\end{gathered}
$$

The Lagrangian (3.1) in the elliptic coordinates is determined by the formulae $[2,6]$,

$$
L=\frac{1}{2} \sum_{j=0}^{n} g_{j} \dot{u}_{j}^{2}-V, \quad g_{j}=\frac{1}{4} \frac{U_{0}^{\prime}\left(u_{j}\right)}{A\left(u_{j}\right)} .
$$

The corresponding Hamiltonian system and the Hamiltonian $H$ look as follows

$$
\begin{gathered}
\dot{p}_{j}=-\partial H / \partial u_{j}, \quad \dot{u}_{j}=\partial H / \partial p_{j}, \\
H\left(p_{j}, u_{j}\right)=\frac{1}{2} \sum_{j=0}^{n} g_{j}^{-1} p_{j}^{2}+V, \quad p_{j}=g_{j} \dot{u}_{j} .
\end{gathered}
$$

The Hamilton-Jacobi equation for the generating function $S\left(u_{0}, \ldots, u_{n}, \eta_{0}, \ldots, \eta_{n}\right)$ has the form

$$
\sum_{j=0}^{n} \frac{2 A\left(u_{j}\right)}{U_{0}^{\prime}\left(u_{j}\right)}\left(\frac{\partial S}{\partial u_{j}}\right)^{2}+V=\eta_{0} .
$$

Proposition 1. The Hamilton-Jacobi Eq. (3.7) and the Hamiltonian Eqs. (3.6) are integrable in quadratures if the potential $V(u)$ is defined by the formula

$$
V(u)=\frac{1}{2 \pi i} \oint \frac{f(z) z^{n+1}}{U_{0}(z)} d z=\sum \operatorname{Res} \frac{f(z) z^{n+1}}{U_{0}(z)},
$$

where $f(z)$ is an arbitrary entire function of $z$ with constant real coefficients and the integral is taken around an infinitely distant circle in the complex plane. The potential $V(u)$ is the entire function of the symmetric variables $\sigma_{1}, \ldots, \sigma_{n+1}$ and the Euclidean coordinates $x_{0}, \ldots, x_{n}$. If the function $f(z)$ is a polynomial of degree $N$, then the potential $V(x)$ in the coordinates $x_{0}, \ldots, x_{n}$ is a polynomial of degree $2 N+2$.

We introduce the polynomial $P_{n}(z)=\eta_{0} z^{n}+\ldots+\eta_{n}$. From the residue theorem one receives

$$
\eta_{0}=\frac{1}{2 \pi i} \oint \frac{P_{n}(z)}{U_{0}(z)} d z=\sum_{j=0}^{n} \frac{P_{n}\left(u_{j}\right)}{U_{0}^{\prime}\left(u_{j}\right)} .
$$


Analogously for the potential (3.8) one obtains the representation

$$
V(u)=\sum_{j=0}^{n} \frac{f\left(u_{j}\right) u_{j}^{n+1}}{U_{0}^{\prime}\left(u_{j}\right)} .
$$

In consequence of this formulae the Hamilton-Jacobi Eq. (3.7) acquires the form

$$
\sum_{j=0}^{n} \frac{1}{U_{0}^{\prime}\left(u_{j}\right)}\left(2 A\left(u_{j}\right)\left(\frac{\partial S}{\partial u_{j}}\right)^{2}+f\left(u_{j}\right) u_{j}^{n+1}-P_{n}\left(u_{j}\right)\right)=0 .
$$

Equation (3.9) has the following solution:

$$
S\left(u_{0}, \ldots, u_{n}, \eta_{0}, \ldots, \eta_{n}\right)=\sum_{j=0}^{n} \int_{0}^{u_{j}}\left(\frac{f(z) z^{n+1}-P_{n}(z)}{-2 A(z)}\right)^{1 / 2} d z .
$$

The generating function (3.10) determines the canonical transformation by the following formulae

$$
\begin{gathered}
p_{j}=\frac{\partial S}{\partial u_{j}}=\left(\frac{f\left(u_{j}\right) u_{j}^{n+1}-P_{n}\left(u_{j}\right)}{-2 A\left(u_{j}\right)}\right)^{1 / 2}, \\
\xi_{k}=-\frac{\partial S}{\partial \eta_{k}}=\frac{1}{2} \sum_{j=0}^{n} \int_{0}^{u_{j}} \frac{z^{n-k} d z}{\left(-2 A(z)\left(f(z) z^{n+1}-P_{n}(z)\right)\right)^{1 / 2}} .
\end{gathered}
$$

As follows from Eq. (3.7) the Hamiltonian Eqs. (3.6) in the canonical coordinates $\xi_{k}, \eta_{k}$ have the form

$$
\dot{\xi}_{k}=-\delta_{k 0}, \dot{\eta}_{k}=0, \quad \xi_{k}(t)=-t \delta_{k 0}+c_{k} .
$$

Therefore the dynamics of trajectories in the coordinates $p_{j}, u_{j}$ is integrable in quadratures after inversion of the transformation determined by Eqs. (3.11).

One can find the expressions of the first integrals in terms of the variables $p_{j}, u_{j}$ from the system of linear equations,

$$
\eta_{0} u_{j}^{n}+\eta_{1} u_{j}^{n-1}+\ldots+\eta_{n}=f\left(u_{j}\right) u_{j}^{n+1}+2 A\left(u_{j}\right) p_{j}^{2}, \quad j=0, \ldots, n .
$$

The integrals $\eta_{k}$ are quadratic functions of the momenta $p_{j}$.

To prove the last part of the Proposition 1, we use the formula

$$
\begin{gathered}
\frac{f(z) z^{n+1}}{U_{0}(z)}=\frac{f(z)}{1-Z}=f(z)\left(1+Z+Z^{2}+\ldots\right), \\
Z=1-\prod_{j=0}^{n}\left(1-\frac{u_{j}}{z}\right)=\sum_{k=1}^{n+1}(-1)^{k+1} \frac{\sigma_{k}\left(u_{0}, \ldots, u_{n}\right)}{z^{k}} .
\end{gathered}
$$

Assuming in the integral (3.8) $|z| \gg 1$ one obtains that the potential $V(u)$ is the entire function of the variables $\sigma_{1}, \ldots, \sigma_{n+1}$ and coincides with the coefficient at $z^{-1}$ in the Laurent expansion determined by Eqs. (3.12). Hence we find that if the function $f(z)$ is a polynomial $f(z)=c_{0}+c_{1} z+\ldots+c_{N} z^{N}$, then the potential $V(u)$ is also a polynomial and looks as follows:

$$
\begin{aligned}
V(u)= & c_{0} \sigma_{1}+c_{1}\left(\sigma_{1}^{2}-\sigma_{2}\right)+c_{2}\left(\sigma_{1}^{3}-2 \sigma_{1} \sigma_{2}+\sigma_{3}\right) \\
& +c_{3}\left(\sigma_{1}^{4}-3 \sigma_{1}^{2} \sigma_{2}+2 \sigma_{1} \sigma_{3}+\sigma_{2}^{2}-\sigma_{4}\right)+\ldots+c_{N}\left(\sigma_{1}^{N+1}-N \sigma_{1}^{N-1} \sigma_{2}+\ldots\right) .
\end{aligned}
$$


One can obtain the form of the potential in the Cartesian coordinates $x_{0}, \ldots, x_{n}$ from Eqs. (3.12)-(3.13) after a substitution of the expressions (3.3) of the symmetric functions $\sigma_{k}(u)$ in terms of the second-order polynomials $Q_{k-1}(x)$. In [19] a quartic potential was pointed out for which the problem of the dynamics of $n$ interacting particles on a straight line has $n$ involutive first integrals. This potential may be included in the family of the potentials (3.13) as the simplest case determined by the conditions $c_{k}=0$ for all $k \geqq 2$.

Evidently the second-order terms in the two first summands in the polynomials (3.13) [after a substitution of the expressions (3.3)-(3.4)] can be made arbitrary (diagonal) by choosing the constants $a_{0}, \ldots, a_{n}, c_{0}, c_{1}$ in such a way that the coefficient $c_{1}$ is arbitrary small. Therefore if the coefficients $c_{2}, \ldots, c_{N}$ are also small, the potentials (3.13) describe the integrable perturbations of degree $2 N+2$ for the system of $n+1$ harmonic oscillators on a straight line. The terms of the greatest degree in the polynomials $V_{2 N+2}\left(x_{0}, \ldots, x_{n}\right)$ have the form

$$
c_{N}\left(x_{0}^{2}+x_{1}^{2}+\ldots+x_{n}^{2}\right)^{N+1} .
$$

The found class of the "integrable" potentials $V(x)$ (3.8) [in particular the polynomials (3.13)] is closed under addition.

2. We consider a family of integrable Lagrangian systems with the Lagrangians of the form (3.1) on the ellipsoid defined by the equation

$$
\sum_{v=0}^{n} \frac{x_{v}^{2}}{a_{v}}=1, \quad a_{v} \neq a_{\mu}>0 .
$$

In the elliptic coordinates (3.2), Eq. (3.14) acquires the form $u_{0}=0$. Therefore the function $U_{0}(z)$ on the ellipsoid takes the form

$$
U_{0}(z)=z U(z), \quad U(z)=\prod_{j=1}^{n}\left(z-u_{j}\right) .
$$

The Lagrangian (3.1) on the ellipsoid (3.14) in the elliptic coordinates (3.2) has the form (3.5), where $j=1, \ldots, n$. The Hamilton-Jacobi Eq. (3.7) acquires the form

$$
\sum_{j=1}^{n} \frac{2 A\left(u_{j}\right)}{u_{j} U^{\prime}\left(u_{j}\right)}\left(\frac{\partial S}{\partial u_{j}}\right)^{2}+V=\eta_{1} .
$$

Proposition 2. The Hamilton-Jacobi Eq. (3.16) and the corresponding Lagrangian system on the ellipsoid (3.14) are integrable in quadratures if the potential $V(u)$ is determined by the formula

$$
V(u)=\frac{1}{2 \pi i} \oint \frac{f(z) z^{n}}{U(z)} d z=\sum \operatorname{Res} \frac{f(z) z^{n}}{U(z)},
$$

where $f(z)$ is an arbitrary entire function with real coefficients. If $f(z)$ is a polynomial of degree $N$, then the potential $V(x)$ in the Cartesian coordinates $x_{0}, \ldots, x_{n}$ is a polynomial of degree $2 N+2$.

The proof is similar to that above. The polynomial $P_{n}(z)$ is replaced by the polynomial $P_{n-1}(z)=\eta_{1} z^{n-1}+\eta_{2} z^{n-2}+\ldots+\eta_{n}$. The generating function $S(u, \eta)$ has the form

$$
S\left(u_{1}, \ldots, u_{n}, \eta_{1}, \ldots, \eta_{n}\right)=\sum_{j=1}^{n} \int_{0}^{u_{j}}\left(\frac{z\left(f(z) z^{n}-P_{n-1}(z)\right)}{-2 A(z)}\right)^{1 / 2} d z
$$


The final formulae for the "integrable" potentials $V\left(x_{0}, \ldots, x_{n}\right)$, Eqs. (3.13)-(3.3), remain valid without any changes (at $u_{0}=0$ ).

For $f(z) \equiv 0(V(x) \equiv 0)$ the generating function (3.18) defines the integration of the geodesic flow on the ellipsoid [2,3]. For $f(z) \equiv 1$ we have from Eq. (3.13),

$$
V_{2}\left(x_{0}, \ldots, x_{n}\right)=x_{0}^{2}+x_{1}^{2}+\ldots+x_{n}^{2}-\sigma_{1}(a) .
$$

This integrable case was also found by Jacobi [2]. For $f(z)=c_{0}+c_{1} z$, we obtain from Eqs. 3.13)-3.3) the quartic potential

$$
V_{4}\left(x_{0}, \ldots, x_{n}\right)=c_{1} V_{2}^{2}+c_{0} V_{2}-c_{1}\left(\sigma_{2}(a)-\sigma_{1}(a) \sum_{v=0}^{n} x_{v}^{2}-\sum_{v=0}^{n} a_{v} x_{v}^{2}\right),
$$

which determine integrable dynamics of a mass point on the ellipsoid (3.14). The polynomial potentials $V_{2 N+2}(x)(3.13)$-(3.3) determine the infinite-dimensional set of integrable cases of dynamics of a mass point on the ellipsoid (3.14).

3. For construction of integrable Lagrangian systems on the sphere $S^{n}$, defined by the equation $Q_{0}(x)=x_{0}^{2}+x_{1}^{2}+\ldots+x_{n}^{2}=1$, the special elliptic coordinates $u_{1}, \ldots, u_{n}[2,6]$ are useful, which are determined from the equation

$$
\sum_{v=0}^{n} \frac{x_{v}^{2}}{z+a_{v}}=\frac{U(z)}{A(z)}, U(z)=\prod_{j=1}^{n}\left(z-u_{j}\right), A(z)=\prod_{v=0}^{n}\left(z+a_{v}\right) .
$$

After reduction to the common denominator $A(z)$ and equating the coefficients at $z^{n-k}(k=0, \ldots, n)$ in both-hand sides of Eq. (3.21), one obtains the relations

$$
\sigma_{k}(u)=(-1)^{k} Q_{k}\left(x_{0}, \ldots, x_{n}\right) .
$$

The Lagrangian (3.1) in the elliptic coordinates (3.21) on the sphere $S^{n}$ is determined by the formula [6],

$$
L=\frac{1}{2} \sum_{j=1}^{n} h_{j} \dot{u}_{j}^{2}-V, \quad h_{j}=-\frac{1}{4} \frac{U^{\prime}\left(u_{j}\right)}{A\left(u_{j}\right)} .
$$

The corresponding Hamilton-Jacobi equation has the form

$$
-\sum_{j=1}^{n} \frac{2 A\left(u_{j}\right)}{U^{\prime}\left(u_{j}\right)}\left(\frac{\partial S}{\partial u_{j}}\right)^{2}+V=\eta_{1}
$$

This equation is analogous to Eqs. (3.7), (3.16). Therefore Proposition 2 is completely applicable also for the integrable Lagrangian systems construction on the sphere $S^{n}$, the potential $V(u)$ is determined by the formula (3.17). The generating function $S(u, \eta)$ has the form

$$
S\left(u_{1}, \ldots, u_{n}, \eta_{1}, \ldots, \eta_{n}\right)=\sum_{j=1}^{n} \int_{0}^{u_{j}}\left(\frac{f(z) z^{n}-P_{n-1}(z)}{2 A(z)}\right)^{1 / 2} d z .
$$

After the canonical transformation $p_{j}, u_{j} \rightarrow \xi_{k}, \eta_{k}\left(p_{i}=h_{i} \dot{u}_{i}\right)$ determined as follows

$$
\begin{gathered}
p_{j}=\frac{\partial S}{\partial u_{j}}=\left(\frac{f\left(u_{j}\right) u_{j}^{n}-P_{n-1}\left(u_{j}\right)}{2 A\left(u_{j}\right)}\right)^{1 / 2}, \\
\xi_{k}=-\frac{\partial S}{\partial \eta_{k}}=\frac{1}{2} \sum_{j=1}^{n} \int_{0}^{u_{j}} \frac{z^{n-k} d z}{\left(2 A(z)\left(f(z) z^{n}-P_{n-1}(z)\right)\right)^{1 / 2}},
\end{gathered}
$$


the Hamiltonian system [corresponding to the Lagrangian (3.23)] takes the simplest form

$$
\dot{\xi}_{k}=-\delta_{k 1}, \quad \dot{\eta}_{k}=0, \quad \xi_{k}(t)=-t \delta_{k 1}+c_{k} .
$$

Therefore the dynamics in the variables $p_{j}, u_{j}$ is integrable in quadratures after inversing the transformation determined by Eqs. (3.26). The formulae for the first integrals $\eta_{k}$ in terms of the variables $p_{j}, u_{j}$ can be obtained from the system of linear equations

$$
\eta_{1} u_{j}^{n-1}+\eta_{2} u_{j}^{n-2}+\ldots+\eta_{n}=f\left(u_{j}\right) u_{j}^{n}-2 A\left(u_{j}\right) p_{j}^{2}, \quad j=1, \ldots, n .
$$

The form of the "integrable" potentials $V\left(x_{0}, \ldots, x_{n}\right)$ in the Cartesian coordinates $x_{0}, \ldots, x_{n}$ is determined by Eqs. (3.12), (3.13), where instead of $\sigma_{k}(u)$ it is necessary to substitute, according to Eq. (3.22), the polynomials $(-1)^{k} Q_{k}(x)$.

The classification of the two-dimensional natural Lagrangian systems integrable by the Hamilton-Jacobi method was done in [20]. The class of the "integrable" potentials $V\left(u_{1}, \ldots, u_{n}\right)$ on the spheres $S^{n}$ in the special case $n=2$ coincides with the "integrable" potentials on the two-dimensional sphere $S^{2}$, found in [21], where their explicit forms were received in so-called "spherical-conical" coordinates. We indicate the formulae for these potentials in terms of the Cartesian coordinates $x_{0}, x_{1}, x_{2}$, which follows from Eqs. (3.12), (3.13) at $n=2$,

$$
\begin{gathered}
V\left(x_{0}, x_{1}, x_{2}\right)=\sum_{N=1}^{\infty} \sum_{k=0}^{N}(-1)^{k} c_{N+k-1} C_{N}^{k} \sigma_{1}^{N-k} \sigma_{2}^{k}, \\
\sigma_{1}(u)=-\left(a_{0}+a_{1}+a_{2}\right)+\sum_{v=0}^{2} a_{v} x_{v}^{2}, \\
\sigma_{2}(u)=a_{0} a_{1}+a_{0} a_{2}+a_{1} a_{2}-\left(a_{0}+a_{1}+a_{2}\right) \sum_{v=0}^{2} a_{v} x_{v}^{2}+\sum_{v=0}^{2} a_{v}^{2} x_{v}^{2} .
\end{gathered}
$$

Here $C_{N}^{k}=N ! / k !(N-k)$ !, the constants $c_{k}$ satisfy the condition that the series $f(z)=\sum_{m=0}^{\infty} c_{m} z^{m}$ is convergent at all values of $z$.

Remark 1. The separation of variables for the Lagrangian (3.1) in the elliptic coordinates is possible if the potential $V(u)$ has the form

$$
V\left(u_{0}, \ldots, u_{n}\right)=\sum_{j=0}^{n} \frac{F_{j}\left(u_{j}\right)}{U_{0}^{\prime}\left(u_{j}\right)} .
$$

However the formulae for the potentials (3.28) in the Cartesian coordinates $x_{0}, \ldots, x_{n}$ include radicals, in contrast with the potentials (3.8), (3.17) which are analytical functions in Cartesian coordinates and include the polynomials of all even degrees.

\section{Integrable Cases of the Equations of a Rigid Body Rotation in an Axially Symmetric Potential Field}

Rotation of a rigid body around a fixed point in an axially symmetric (for example central) potential field is described by the Lagrangian system on the Lie group 
$\mathrm{SO}(3)$ with the Lagrangian,

$$
L=\frac{1}{2}\left(I_{1} \omega_{1}^{2}+I_{2} \omega_{2}^{2}+I_{3} \omega_{3}^{2}\right)-V(\mathbf{q})
$$

where the "Poisson vector" $\mathbf{q}(t)$ has the following components (in the rotating frame $S$ )

$$
q_{1}=\sin \theta \sin \varphi, \quad q_{2}=\sin \theta \cos \varphi, \quad q_{3}=\cos \theta .
$$

The angular velocities $\omega_{1}, \omega_{2}, \omega_{3}$ have the following expressions in terms of the Euler angles $\theta, \varphi, \psi[1]$ :

$$
\begin{gathered}
\omega_{1}=\dot{\psi} \sin \theta \sin \varphi+\dot{\theta} \cos \varphi, \quad \omega_{2}=\dot{\psi} \sin \theta \cos \varphi-\dot{\theta} \sin \varphi, \\
\omega_{3}=\dot{\psi} \cos \theta+\dot{\varphi} .
\end{gathered}
$$

The precession angle $\psi$ is the cyclic coordinate for the Lagrangian (4.1). The corresponding momentum $p_{\psi}$ is the first integral of the Lagrangian system

$$
p_{\psi}=\partial L / \partial \dot{\psi}=I_{1} \omega_{1} q_{1}+I_{2} \omega_{2} q_{2}+I_{3} \omega_{3} q_{3}=(\mathbf{M}, \mathbf{q}) \text {. }
$$

The Lagrange equations (4.1) in terms of the coordinates of the angular momentum vector $\mathbf{M}$ and the Poisson vector $\mathbf{q}$ are as follows:

$$
\dot{\mathbf{M}}=\mathbf{M} \times \boldsymbol{\omega}+\mathbf{q} \times \partial V / \partial \mathbf{q}, \quad \dot{\mathbf{q}}=\mathbf{q} \times \boldsymbol{\omega} .
$$

Equations (4.5) for a constant value of the first integral $(\mathbf{M}, \mathbf{q})=p_{\psi}=$ const are the Lagrangian equations on the two-dimensional sphere $S^{2}$, their Lagrangian is the Routh function (this fact was first noted in [22] and was used for the proof of the existence of closed trajectories in $[23,24]$ ) and has the form

$$
\begin{aligned}
L_{1}= & L-p_{\psi} \dot{\psi}=\frac{1}{2} \dot{\theta}^{2}\left(I_{1} \cos ^{2} \varphi+I_{2} \sin ^{2} \varphi\right)+\frac{1}{2} I_{3} \dot{\varphi}^{2} \\
& -\frac{\left(\left(I_{1}-I_{2}\right) \dot{\theta} \sin \theta \sin \varphi \cos \varphi+I_{3} \dot{\varphi} \cos \theta-p_{\psi}\right)^{2}}{2 \sin ^{2} \theta\left(I_{1} \sin ^{2} \varphi+I_{2} \cos ^{2} \varphi\right)+2 I_{3} \cos ^{2} \theta}-V(\theta, \varphi) .
\end{aligned}
$$

Minkowski noted the geometrical sense of the Lagrangian $L_{1}$.

Geometric Lemma. The Lagrangian $L_{1}$ (4.6) for $p_{\psi}=0$ describes a mass point motion under the influence of the forces with the potential $V(\theta, \varphi)$ on the surface of the inertia ellipsoid

$$
I_{1} x_{1}^{2}+I_{2} x_{2}^{2}+I_{3} x_{3}^{2}=1
$$

in the metric, conformally equivalent to the standard one and having the form [22]

$$
d s^{2}=\frac{I_{1} I_{2} I_{3}\left(d x_{1}^{2}+d x_{2}^{2}+d x_{3}^{2}\right)}{I_{1}^{2} x_{1}^{2}+I_{2}^{2} x_{2}^{2}+I_{3}^{2} x_{3}^{2}} .
$$

We introduce the angular coordinates $\theta, \varphi$ on the ellipsoid (4.7),

$$
x_{1}=I_{1}^{-1 / 2} \sin \theta \sin \varphi, \quad x_{2}=I_{2}^{-1 / 2} \sin \theta \cos \varphi, \quad x_{3}=I_{3}^{-1 / 2} \cos \theta .
$$


The kinetic energy of the mass point, corresponding to the metric (4.8), acquires the following form in the coordinates $\theta, \varphi$ :

$$
\begin{aligned}
T= & {\left[2 \sin ^{2} \theta\left(I_{1} \sin ^{2} \varphi+I_{2} \cos ^{2} \varphi\right)+2 I_{3} \cos ^{2} \theta\right]^{-1} } \\
& \cdot\left[\dot{\varphi}^{2} I_{3} \sin ^{2} \theta\left(I_{1} \sin ^{2} \varphi+I_{2} \cos ^{2} \varphi\right)+\dot{\theta}^{2}\left(I_{1} I_{2} \sin ^{2} \theta+I_{3} \cos ^{2} \theta\right.\right. \\
& \left.\left.\cdot\left(I_{1} \cos ^{2} \varphi+I_{2} \sin ^{2} \varphi\right)\right)-2 \dot{\theta} \dot{\varphi} I_{3}\left(I_{1}-I_{2}\right) \sin \theta \cos \theta \sin \varphi \cos \varphi\right] .
\end{aligned}
$$

One can verify by direct calculation the kinetic energy (4.10) coincides with the kinetic energy (4.6) for $p_{\psi}=0$. This fact completes the proof of the Geometric Lemma because of the identity of two potentials $V(\theta, \varphi)$ in Eqs. (4.6), (4.8).

Due to the Geometric Lemma the kinetic energy (4.10) coincides with the Routh function for the Euler rotation of a free rigid body for $p_{\psi}=0$. Therefore it is obvious that the geodesic flow for the metric (4.8) is integrable in the elliptic functions. The integrability in Liouville's sense was proved in [25] for the geodesic flow for the metric of the form (4.8) in $n$-dimensional case.

We determine the elliptic coordinates $u_{1}, u_{2}$ on the ellipsoid (4.7) satisfying the equation

$$
\sum_{v=1}^{3} \frac{x_{v}^{2}}{z+I_{v}^{-1}}-1=-\frac{U(z)}{A(z)}, U(z)=\prod_{i=0}^{2}\left(z-u_{i}\right), A(z)=\prod_{v=1}^{3}\left(z+I_{v}^{-1}\right) .
$$

We have $u_{0}=0$ on the ellipsoid (4.7). If we differentiate (4.11) with respect to $z$ and substitute $z=0$ we obtain for $u_{0}=0$,

$$
I_{1}^{2} x_{1}^{2}+I_{2}^{2} x_{2}^{2}+I_{3}^{2} x_{3}^{2}=I_{1} I_{2} I_{3} u_{1} u_{2} .
$$

Therefore the Lagrangian (4.6) for $p_{\psi}=0$ in consequence of the Geometric Lemma acquires the form

$$
\begin{gathered}
L_{1}=\frac{1}{2 u_{1} u_{2}}\left(g_{1} \dot{u}_{1}^{2}+g_{2} \dot{u}_{2}^{2}\right)-V\left(u_{1}, u_{2}\right), \\
g_{1}=\frac{u_{1}\left(u_{1}-u_{2}\right)}{4 A\left(u_{1}\right)}, \quad g_{2}=-\frac{u_{2}\left(u_{1}-u_{2}\right)}{4 A\left(u_{2}\right)} .
\end{gathered}
$$

We suppose the potential $V\left(u_{1}, u_{2}\right)$ to be of the form

$$
V\left(u_{1}, u_{2}\right)=u_{1} u_{2} \frac{F_{1}\left(u_{1}\right)-F_{2}\left(u_{2}\right)}{u_{1}-u_{2}}
$$

We introduce the momenta $p_{1}=g_{1} \dot{u}_{1} / u_{1} u_{2}, p_{2}=g_{2} \dot{u}_{2} / u_{1} u_{2}$. The Hamiltonian $H(p, u)$ corresponding to the Lagrangian $L_{1}$, is as follows:

$$
H=\frac{1}{u_{2}^{-1}-u_{1}^{-1}}\left[\frac{2 A\left(u_{1}\right)}{u_{1}} p_{1}^{2}-\frac{2 A\left(u_{2}\right)}{u_{2}} p_{2}^{2}+F_{1}\left(u_{1}\right)-F_{2}\left(u_{2}\right)\right],
$$

and belongs to Liouville's type. The Hamilton-Jacobi equation

$$
H\left(\frac{\partial S}{\partial u}, u\right)=\eta_{1}, \quad S=S\left(u_{1}, u_{2}, \eta_{1}, \eta_{2}\right)
$$


has the solution, satisfying two separate equations,

$$
\begin{aligned}
& \frac{2 A\left(u_{1}\right)}{u_{1}}\left(\frac{\partial S}{\partial u_{1}}\right)^{2}+F_{1}\left(u_{1}\right)=-\frac{\eta_{1}}{u_{1}}+\eta_{2}, \\
& \frac{2 A\left(u_{2}\right)}{u_{2}}\left(\frac{\partial S}{\partial u_{2}}\right)^{2}+F_{2}\left(u_{2}\right)=-\frac{\eta_{1}}{u_{2}}+\eta_{2} .
\end{aligned}
$$

This solution has the form

$$
S(u, \eta)=\int_{0}^{u_{1}}\left(\frac{z \eta_{2}-\eta_{1}-z F_{1}(z)}{2 A(z)}\right)^{1 / 2} d z+\int_{0}^{u_{2}}\left(\frac{z \eta_{2}-\eta_{1}-z F_{2}(z)}{2 A(z)}\right)^{1 / 2} d z .
$$

The canonical transformation $p_{j}, u_{j} \rightarrow \xi_{k}, \eta_{k}$, defined by the formulae

$$
p_{k}=\frac{\partial S}{\partial u_{k}}, \quad \xi_{k}=-\frac{\partial S}{\partial \eta_{k}}, \quad k=1,2,
$$

transforms the Hamiltonian flow under study to the simplest flow

$$
\xi_{1}=-t+c_{1}, \quad \xi_{2}=c_{2}, \quad \eta_{1}=c_{3}, \quad \eta_{2}=c_{4} .
$$

Thus the following theorem is proved.

Theorem 1. The equations of a rigid body rotation around a fixed point in an axially symmetric potential field are completely integrable in Liouville's sense for $p_{\psi}=0$ if the potential function has the form (4.13), where $F_{1}(u), F_{2}(u)$ are arbitrary smooth functions. The explicit integration is reduced to the quadratures by the HamiltonJacobi method.

The additional integral $\eta_{2}$ has the form

$$
\eta_{2}=\frac{u_{1}-u_{2}}{16}\left(\frac{\dot{u}_{1}^{2}}{u_{2}^{2} A\left(u_{1}\right)}-\frac{\dot{u}_{2}^{2}}{u_{1}^{2} A\left(u_{2}\right.}\right)+\frac{u_{1} F_{1}\left(u_{1}\right)-u_{2} F_{2}\left(u_{2}\right)}{2\left(u_{1}-u_{2}\right)} .
$$

In the special case $F_{1}(z)=F_{2}(z)=f(z)$ [35] (the series $f(z)=\sum_{m=0}^{\infty} c_{m} z^{m}$ defines the entire function) the potential (4.13) is determined also by the formula

$$
V\left(u_{1}, u_{2}\right)=\frac{u_{1} u_{2}}{2 \pi i} \oint \frac{f(z) d z}{\left(z-u_{1}\right)\left(z-u_{2}\right)},
$$

and is the entire function of the Cartesian coordinates $x_{1}, x_{2}, x_{3}$ (see Sect. 3). In consequence of Eq. (4.11) we have

$$
\begin{aligned}
& \sigma_{1}=u_{1}+u_{2}=x_{1}^{2}+x_{2}^{2}+x_{3}^{2}-\left(I_{1}^{-1}+I_{2}^{-1}+I_{3}^{-1}\right), \\
& \sigma_{2}=u_{1} u_{2}=\left(I_{1} I_{2} I_{3}\right)^{-1}\left(I_{1}^{2} x_{1}^{2}+I_{2}^{2} x_{2}^{2}+I_{3}^{2} x_{3}^{2}\right) .
\end{aligned}
$$

The potential (4.19) in the coordinates $x_{1}, x_{2}, x_{3}$ takes the form [see Eq. (3.12)]

$$
V\left(x_{1}, x_{2}, x_{3}\right)=\sigma_{2}\left(c_{0}+\sum_{N=1}^{\infty} \sum_{k=0}^{N}(-1)^{k} c_{N+k-1} C_{N}^{k} \sigma_{1}^{N-k} \sigma_{2}^{k}\right) .
$$


After a substitution of the relations $x_{k}=q_{k} I_{k}^{-1 / 2}$ one obtains the expressions of the potentials (4.21) in terms of the Poisson vector coordinates $q_{k}$. If $f(z)=c_{0}+c_{1} z$, then the function (4.21) is the potential $c_{1}\left(I_{1} q_{1}^{2}+I_{2} q_{2}^{2}+I_{3} q_{3}^{2}\right)$ of the Brun integrable case [26], coinciding with the Clebsch integrable case [12] for the Kirchhoff equations. For $f(z)=c_{0}+c_{1} z+c_{2} z^{2}$, we obtain an integrable case, corresponding to the potential

$$
V_{4}=\left(I_{1} q_{1}^{2}+I_{2} q_{2}^{2}+I_{3} q_{3}^{2}\right)\left(c+c_{2}\left(I_{1}^{-1} q_{1}^{2}+I_{2}^{-1} q_{2}^{2}+I_{3}^{-1} q_{3}^{2}\right)\right) .
$$

If the function $f(z)$ is a polynomial of degree $N$, then the corresponding potential $V\left(q_{1}, q_{2}, q_{3}\right)$ (4.21) is the polynomial of degree $2 N$. The whole family of "integrable" potentials (4.21) depends on the infinite set of parameters $c_{k}$ and is closed with respect to the addition.

\section{Integrable Cases of the Equations of a Rigid Body Rotation with a Symmetric and Spherical Inertia Tensor}

1. The Lagrangian describing rotation of a symmetric $\left(I_{1}=I_{2}\right)$ rigid body around a fixed point has the form (2.3). We suppose the potential function depends on two Euler angles $V=V(\theta, \psi)$. Then the coordinate $\varphi$ is cyclic and the corresponding momentum $p_{\varphi}$ (2.4) is the first integral. Let $\mathbf{q}(t)$ be the unit vector along the symmetry axes of the rigid body inertia tensor, with the coordinates (2.2). The Routh function $L_{2}=L_{1}-p_{\varphi} \dot{\varphi}$ has the form

$$
L_{2}=\frac{1}{2} I_{1}(\dot{\mathbf{q}}, \dot{\mathbf{q}})+p_{\varphi} \dot{\psi} \cos \theta-V(\mathbf{q})-\frac{1}{2 I_{3}} p_{\varphi}^{2} .
$$

The Lagrangian (5.1) for $p_{\varphi}=0$ describes the dynamics of a mass point on the sphere $S^{2}$ in the potential field with the potential $V(\mathbf{q})$. Thus to every integrable case of this problem there corresponds an integrable case of the symmetric rigid body dynamics for $p_{\varphi}=0$.

Corollary. The equations of a symmetric rigid body rotation around a fixed point in a potential field are integrable in Liouville's sense for $p_{\varphi}=0$ if the potential function $V(\theta, \psi)$ belongs to the infinite-dimensional set of the potentials (3.27), where

$$
x_{0}=\cos \theta, \quad x_{1}=\sin \theta \sin \psi, \quad x_{2}=-\sin \theta \cos \psi .
$$

The dynamics is integrable in quadratures.

Remark 2. Two maps $\mathrm{SO}(3) \rightarrow S^{2}$ used in the constructions of the integrable cases here and in Sect. 4 are essentially different. In Sect. 4 the factorization is used with respect to the precession angle $\psi$, but in the construction pointed out above (and in Sect. 2) the factorization is realized with respect to the angle of the proper rotation $\varphi$.

Applications of the fibration $S^{3} \rightarrow S^{2}$ to the Kepler's problem were discovered in [32].

2. The covering map

$$
f: S^{3} \rightarrow \mathrm{SO}(3)
$$


is determined by the formulae [27]

$$
Q_{j}^{i}\left(x_{0}, x_{1}, x_{2}, x_{3}\right)=2\left(x_{i} x_{j}+\left(x_{0}^{2}-\frac{1}{2}\right) \delta_{i j}-x_{0} x_{k} \varepsilon_{i j k}\right) .
$$

The map (5.2)-(5.3) is also the homomorphism of the Lie groups (the sphere $S^{3}$ is the Lie group of quaternions of unit norm). Therefore the bilaterally invariant metric on the Lie group $\mathrm{SO}(3)$ corresponding to the spherical inertia tensor transforms under the mapping $f^{*}$ to the bilaterally invariant metric on the sphere $S^{3}$ coinciding with the standard one.

The map (5.2)-(5.3) identifies diametrically opposite points on the sphere $S^{3}$ : $Q_{j}^{i}(\mathbf{x})=Q_{i}^{j}(-\mathbf{x})$. The elliptic coordinates $u_{1}, \ldots, u_{n}(3.21)$ depend on the squares of the coordinates $x_{v}$; thus the same coordinates $u_{1}, \ldots, u_{n}$ correspond to every two diametrically opposite points on the sphere $S^{n}$. Hence the elliptic coordinates on the sphere $S^{3}$ are also the coordinates on the Lie group $\mathrm{SO}(3)$. Therefore every Lagrangian system on the sphere $S^{3}$ with the Lagrangian (3.1) integrable in the elliptic coordinates (3.21) determines an integrable Lagrangian system on the Lie group $\mathrm{SO}(3)$ with the Lagrangian

$$
L_{1}=I_{0} \operatorname{Tr}\left(Q^{-1} \dot{Q} Q^{-1} \dot{Q}\right)-V_{1}\left(Q_{j}^{i}\right)
$$

The Lagrangians (5.4) describe the integrable cases of dynamics of a rigid body with the spherical inertia tensor $\left(I_{i k}=I_{0} \delta_{i k}\right)$ in a potential field which determines the potential function $V_{1}\left(Q_{j}^{i}\right)$.

To obtain the explicit formulae for "integrable" potentials $V_{1}\left(Q_{j}^{i}\right)$ we indicate the analytical form of the two-valued transformation inverse to the covering map $(5.2)-(5.3)$

$$
\begin{array}{cc}
x_{0}^{2}=\frac{1}{4}(1+\operatorname{Tr}(Q)), & x_{i}^{2}=\frac{1}{4}\left(1+2 Q_{i}^{i}-\operatorname{Tr}(Q)\right), \\
x_{i} x_{j}=\frac{1}{4}\left(Q_{j}^{i}+Q_{i}^{j}\right), & x_{0} x_{k}=\frac{1}{4} \varepsilon_{i j k}\left(Q_{i}^{j}-Q_{j}^{i}\right) .
\end{array}
$$

Here $i, j, k=1,2,3, i \neq j \neq k$. Due to Eqs. (5.5) we obtain

$$
R_{k}(Q)=\sum_{\nu=0}^{3} a_{v}^{k} x_{v}^{2}(Q)=A_{k}+\left(\frac{1}{2} a_{0}^{k}-A_{k}\right) \operatorname{Tr} Q+\frac{1}{2} \sum_{\nu=1}^{3} a_{v}^{k} Q_{v}^{v},
$$

where $4 A_{k}=a_{0}^{k}+a_{1}^{k}+a_{2}^{k}+a_{3}^{k}$. Because of Eqs. (3.4), (3.22), (5.4) the symmetric functions $\sigma_{1}(u), \sigma_{2}(u), \sigma_{3}(u)$ are the linear functions of the matrix $Q$ elements,

$$
\begin{gathered}
\sigma_{1}(u)=-\sigma_{1}(a)+R_{1}(Q), \quad \sigma_{2}(u)=\sigma_{2}(a)-\sigma_{1}(a) R_{1}(Q)+R_{2}(Q), \\
\sigma_{3}(u)=-\sigma_{3}(a)+\sigma_{2}(a) R_{1}(Q)-\sigma_{1}(a) R_{2}(Q)+R_{3}(Q) .
\end{gathered}
$$

Let the coefficients $c_{m}$ satisfy the condition that the series $f(z)=\sum_{m=0}^{\infty} c_{m} z^{m}$ is convergent at all values of the complex variable $z$.

Theorem 2. There exists an infinite-dimensional set of the integrable in quadratures Lagrangian systems on the Lie group $\mathrm{SO}(3)$ with the Lagrangians (5.4), where the potentials $V_{1}\left(Q_{j}^{i}\right)$ have the form

$$
V_{1}\left(Q_{j}^{i}\right)=\sum_{N=1}^{\infty} \sum_{k=0}^{N} \sum_{\ell=0}^{k}(-1)^{\ell} c_{3 N-2 k+\ell-1} C_{N}^{k} C_{k}^{\ell} \sigma_{1}^{k-\ell} \sigma_{2}^{\ell} \sigma_{3}^{N-k} .
$$


Here $\sigma_{1}, \sigma_{2}, \sigma_{3}$ are the linear functions (5.7) of the matrix $Q$ elements, $C_{N}^{k}=N ! / k !(N-k) !$. The series (5.8) determine the entire function of the matrix $Q$ elements. If the function $f(z)$ is a polynomial of degree $N$, then the potential $V_{1}(Q)$ is the polynomial of degree $N+1$.

The potential $V_{1}(Q)(5.8)$ is the coefficient at $z^{-1}$ in the Laurent expansion of the function (3.12), where

$$
Z=\frac{\sigma_{1}}{z}-\frac{\sigma_{2}}{z^{2}}+\frac{\sigma_{3}}{z^{3}}, \quad n=3
$$

Therefore the proof of Theorem 2 follows from the results of Sect. 3, after application of the transformation (5.2) and the formulae (5.7).

The explicit form of some potentials $V_{1}(Q)$ one can obtain from Eq. (3.13), setting $N=0,1,2,3$. In the simplest case $N=0, f(z)=-c_{0}$, it follows from Theorem 2 that the Lagrangian system (5.4) with the potential

$$
V_{1}\left(Q_{j}^{i}\right)=c_{0}\left(\sigma_{1}(a)-R_{1}(Q)\right)=\frac{3}{4} c_{0} \sigma_{1}(a)+\frac{c_{0}}{4} \sum_{i=1}^{3}\left(a_{0}-a_{1}-a_{2}-a_{3}+2 a_{i}\right) Q_{i}^{i}
$$

is integrable in quadratures.

Note the Lagrangian (5.4) with the potential $V_{1}\left(Q_{j}^{i}\right)$, linear depending on all elements of the matrix $Q: V_{1}(Q)=\sum_{i, j=1}^{3} Q_{j}^{i} B_{i}^{j}$ can be reduced to the one with the potential (5.9) by the transformation $Q=S_{0} Q_{1} T_{0}$, where $S_{0}$ and $T_{0}$ are constant orthogonal matrices. Such transformation preserves the kinetic energy form in Eq. (5.4) and reduces the matrix $B$ to the diagonal form ${ }^{2}$.

We consider a rotation around a fixed point of a magnetized rigid body $T$ with the spherical inertia tensor $\left(I_{i k}=I_{0} \delta_{i k}\right)$ at the presence of an arbitrary distribution of electric charges $\sigma(\mathbf{r})$ satisfying the conditions $(i, j=1,2,3)$

$$
\int_{T}\left(\left(r^{i}\right)^{2}+\left(r^{j}\right)^{2}\right) \sigma(\mathbf{r}) d^{3} \mathbf{r}=0, \quad \int_{T} r^{i} r^{j} \sigma(\mathbf{r}) d^{3} \mathbf{r}=0 .
$$

Theorem 3. The equations of the rigid body $T$ rotation in arbitrary constant gravitational, electric and magnetic fields are completely integrable in Liouville's sense. The dynamics is integrable in terms of the Riemannian theta-functions of three variables $\theta\left(z_{1}, z_{2}, z_{3}\right)$.

At the conditions (5.10) the Lorentz forces momentum equals zero (see [29]), and the equations of the rigid body rotation acquire the form

$$
\dot{\mathbf{M}}=\boldsymbol{\mu} \times Q^{-1} \mathbf{H}+\mathbf{d} \times Q^{-1} \mathbf{E}+m \mathbf{R} \times Q^{-1} \mathbf{G}, \quad \dot{Q}=I_{0}^{-1} Q M,
$$

where $\mathbf{H}, \mathbf{E}, \mathbf{G}$ are the constant (in the resting frame $F$ ) vectors of strengths of the magnetic, electric and gravitational fields, $\boldsymbol{\mu}$ and $\mathbf{d}$ are the vectors of the magnetic and electric dipole momenta of the rigid body, vector $\mathbf{R}$ stands for the position of the center of mass, $m$ is the whole mass of the rigid body (vectors $\boldsymbol{\mu}, \mathbf{d}, \mathbf{R}$ are constant in the rotating frame $S$ ), $M$ is the skew-symmetric matrix corresponding to the angular momentum vector $\mathbf{M}$.

2 The Lax matrix was indicated in [19] for the Lagrangian systems with the linear potentials on the Lie groups $\mathrm{SO}(n)$. Some integrable systems with quartic potentials were found in [28] 
Equations (5.11) are the Lagrangian equations on the Lie group $\mathrm{SO}(3)$ with the Lagrangian

$$
L=\frac{1}{2} I_{0}(\mathbf{M}, \mathbf{M})-\sum_{i, j=1}^{3} Q_{j}^{i} B_{i}^{j}, \quad B_{i}^{j}=\text { const }
$$

The transformation $f$ (5.2)-(5.3) takes the Lagrangian (5.12) into the Lagrangian

$$
f^{*} L=\frac{1}{2} I_{0}\left(\dot{x}_{0}^{2}+\dot{x}_{1}^{2}+\dot{x}_{2}^{2}+\dot{x}_{3}^{2}\right)-\sum_{i, j=0}^{3} a_{i j} x_{i} x_{j}
$$

which coincides with the Lagrangian of the Neumann system on the sphere $S^{3}$ (the matrix $a_{i j}$ is reduced to the diagonal form by the transformation $Q=S_{0} Q_{1} T_{0}$ ). The first integrals of the Neumann system [4-7] are invariant under the reflection $\mathbf{x} \rightarrow-\mathbf{x}$; therefore the integrability of the Lagrangian system with the Lagrangian (5.11) in Liouville's sense follows from that for the Neumann system. As noted above the elliptic coordinates on the sphere $S^{3}$ are also the coordinates on the Lie group SO(3). Therefore the integrability of the rigid body dynamics in terms of the Riemannian theta-functions also follows from that for the Neumann system [4-7].

3. Due to the existence of the local homeomorphism (5.2)-(5.3) it is possible to construct from every integrable Lagrangian system on the Lie group SO(3) some integrable Lagrangian system on the sphere $S^{3}$. On the basis of this construction the integrability was proved [30] for the Lagrangian system on the sphere $S^{3}$ with the Lagrangian (3.1) having the quartic potential

$$
V=\sum_{i \neq j}^{3}\left(x_{i} x_{j}-\varepsilon_{i j k} x_{0} x_{k}\right)^{2} A_{k} J_{j}+\sum_{k=1}^{3}\left(x_{0}^{2}+x_{k}^{2}-\frac{1}{2}\right)^{2} A_{k} J_{k},
$$

where $A_{k}, J_{j}$ are arbitrary constants. Due to the existence of the homomorphism of the Lie groups $S^{3} \times S^{3} \rightarrow \mathrm{SO}(4)$, the integrability was proved [30] for the dynamics of two interacting mass points on the sphere $S^{3}$ (with the standard metric) in the potential field with the quartic potential

$$
\begin{aligned}
V(\mathbf{x}, \mathbf{y})= & \sum_{i \neq j}^{3}\left(x_{i} y_{j}+x_{j} y_{i}-\varepsilon_{i j k}\left(x_{0} y_{k}+y_{0} x_{k}\right)\right)^{2} a_{i} R_{j} \\
& +4 \sum_{k=1}^{3}\left(x_{0} y_{0}+x_{k} y_{k}-\frac{1}{2}(\mathbf{x}, \mathbf{y})\right)^{2} a_{k} R_{k},
\end{aligned}
$$

where $a_{i}, R_{j}$ are arbitrary constants.

Acknowledgements. I would like to thank Prof. D. V. Anosov and Prof. J. Moser for helpful conversations.

\section{References}

1. Leimanis, E.: The general problem of the motion of coupled rigid bodies about a fixed point. Berlin, Heidelberg, New York: Springer 1965 
2. Jacobi, C.G.J.: Vorlesungen über Dynamik, Königsberg 1866

3. Rosochatius, E.: Über Bewegungen eines Punktes (Inaugural Dissertation, University Göttingen). Gebr. Unger, Berlin 1877

4. Neumann, C.: De problemate quodam mechanico, quod ad primam integralium ultraellipticorum classen revocatur. Reine Angew. Math. 56, 46-63 (1859)

5. Moser, J.: Geometry of quadrics and spectral theory. The Chern Symposium, pp. 147-188. Berlin, Heidelberg, New York: Springer 1979

6. Moser, J.: Various aspects of integrable Hamiltonian systems. Progress of Math. Vol. 8. Boston: Birkhäuser 1980; pp. 233-289

7. Moser, J.: Integrable Hamiltonian systems and spectral theory. Lezioni Fermiane. Pisa 1981

8. Knörrer, H.: Geodesics on the ellipsoid. Invent. math. 59, 2, 119-145 (1980)

9. Stackel, P.: Über die Bewegung eines Punktes in einer $n$-fachen Mannigfaltigkeit. Math. Ann. 42, 537-563 (1893)

10. Bogoyavlensky, O.I.: Two integrable cases of a rigid body dynamics in a potential field. Sov. Math. Dokl. 275, 1359-1363 (1984)

11. Bogoyavlensky, O.I.: New integrable problem of classical mechanics. Commun. Math. Phys. 94, 255-269 (1984)

12. Clebsch, A.: Über die Bewegung eines Körpers in einer Flüssigkeit. Math. Ann. 3, 238-262 (1871)

13. Dirac, P.A.M.: The theory of magnetic poles. Phys. Rev. 74, 7, 817-830 (1948)

14. Novikov, S.P., Shmeltser, I.: Periodical solutions of the Kirchhoff equations for free motion of a rigid body in fluid, and the extended Lyusternik-Schnirelman-Morse theory. I. Funct. Anal. Appl. 15, 54-66 (1981)

15. Novikov, S.P.: Hamiltonian formalism and multivalued analogy of the Morse theory. Usp. Mat. Nauk 37, 5, 3-49 (1982)

16. Veselov, A.P.: The Landau-Lifshitz equation and integrable systems of classical mechanics. Sov. Math. Dok1. 270, 5, 1094-1097 (1983)

17. Kotter, F.: Über die Bewegung eines festen Körpers in einer Flüssigkeit. I, II. Reine Angew. Math. 109, 51-81, 89-111 (1892)

18. Steklov, V.A.: On motion of a rigid body in fluid (in Russian). Kharkov: Darre Printing House 1893

19. Reyman, A.G.: Integrable Hamiltonian systems connected with graduated Lie algebras. Zap. Nauchn. Semin. LOMI 95, 3-55 (1980)

20. Morera, G.: Sulla separazione delle variabili. Atti R. Accad. Sci. Torino 16, 276-295 (1881)

21. Eleonsky, V.M., Kulagyn, N.E.: On new cases of integrability for the Landau-Lifshitz equation. ZETP 84, 2, 616-629 (1983)

22. Minkowski, H.: Über die Bewegung eines festen Körpers in einer Flüssigkeit. Sitz. Akad. Wissensch., Berlin 40, 2, 1095-1111 (1888)

23. Arnold, V.I.: Mathematical methods of classical mechanics. Moscow: Nauka 1974

24. Kozlov, V.V.: Methods of qualitative analysis in the rigid body dynamics. Moscow: University Press 1980

25. Brailov, A.V.: Complete integrability of some geodesic flows and the integrable systems with non-commuting integrals. Sov. Math. Dokl. 271, 273-276 (1983)

26. Brun, F.: Rotation kring fix punkt. Ofversigt at Kongl. Svenska Vetenskaps Akademies Forhadlingar, Stockholm 7, 455-468 (1893)

27. Djelobenko, D.P.: Compact Lie groups and their representations. Moscow: Nauka 1970

28. Wojciechowski, S.: Integrability of one particle in a perturbated central quartic potential. Physica Scripta (to appear)

29. Lunev, V.V.: Hydrodynamical analogy for a rigid body motion around a fixed point in the field of Lorentz forces. Sov. Math. Dokl. 276, 351-355 (1984)

30. Bogoyavlensky, O.I.: New integrable problems of classical mechanics. Proc. III Intern. Symp. Selected Probl. Stat. Mechan. Dubna, JINR, 165-170, August 1984

31. Devaney, R.: Transversal homoclinic orbits in an integrable system. Am. J. Math. 100, 631-642 (1978) 
32. Moser, J.: Regularization of Kepler's problem and the averaging method on a manifold. Commun. Pure Appl. Math. 23, 609-636 (1970)

33. Wojciechowski, S.: Two families of integrable one particle potentials which remain integrable when the motion is restricted to a sphere or an ellipsoid. Preprint UMIST, October 1984

34. Wojciechowski, S.: Three families of integrable one particle potentials. Preprint No.440, Universita di Roma, February 1985

35. Kolosov, G.V.: On some alterations of the Hamilton principle. Petersburg: Erlich Printing House 1903

Communicated by Ya. G. Sinai

Received May 10, 1985; in revised form July 8, 1985 